\title{
XXXV. Über Lösungs-, Wachstums- und neutrale Körper aus der Kugel.
}

Von

V. Goldschmidt.

Versuche am Calcit mit Fr. E. Wright ${ }^{1}$ ) und an der Zinkblende mit $\mathbf{P h}$. Hochschild ${ }^{2}$ ) haben gezeigt, daß es zum Studium des Lösungsprocesses, seines Verlaufs und seiner Mechanik gut ist, von der Kugel als Anfangskörper auszugehen. Bei dieser ist durch die Form keine Vorzugsrichtung gegeben und es bleibt der schaffenden Natur überlassen, die Vorzugsrichtungen selbst zu wählen und sie in ihrer eigentümlichen Schrift auf die Oberfläche der Kugel aufzuzeichnen.

Es entstanden Gebilde, die wir Lösungskörper nannten. An ihnen erschienen die Hauptknoten, das sind nach unserer Annahme die Hauptrichtungen der Partikelkräfte des Krystalls, aus dem die Kugel hergestellt ist, zunächst als Ort der Ätzgrübchen, dann als Ecken der Lösungskörper, endlich als der Ort von Bohrlöchern.

Die Hauptzonen erschienen als Reihen von Grübchen, dann als Kanten des Lösungskörpers. Auch aus dem Reflexbild konnte man die Hauptknoten und die Hauptzonen, die Grundzüge des Formensystems der Krystallart herauslesen.

Untersuchungen an Kugeln aus anderem Material, Alaun, Topas (mit Rosick ý) haben gezeigt, was zu erwarten war, daß die gefundenen Erscheinungen allgemeine sind, auch anderen Krystallarten, ja man darf annehmen, all en Krystallarten eigentümlich und daher dazu angetan, über das Wesen der Krystalle und die Mechanik des Lösungsprocesses Aufschluß zu geben.

Neben den Lösungsversuchen an der Kugel gingen solche an Krystallflächen, natürlichen, wie künstlich angeschliffened. Die Versuche ergänzten

1) N. Jahrb. f. Min. 1904, Beil.-Bd. 18, 335; Ausz. diese Zeitschr. 1907, 42, 641.

2) Ebenda 1908, Beil.-Bd. 26, 151; Ausz. diese Zeitschr. 1911, 49, 395. 
sich gegenseitig. Man konnte aus den Kugelerscheinungen auf die Flächenerscheinungen schließen und umgekehrt.

Die Kugel als Anfangskörper ist am geeignetsten. Man kann sie aber nicht aus allen Krystallarten herstellen. Es ist daher wichtig, daß man aus anders gestalteten Anfangskörpern, aus solchen mit Flächen, wie sie die Natur liefert, oder wie sie durch Schleifen hergestellt werden, die entsprechenden Resultate, wenn auch nicht so übersichtlich ableiten kann.

Es lag die Idee nahe, nun auch den WachstumsproceB in seinem Werden bis zum vollen ebenflächigen Krystall, ausgehend von der Kugel, experimentell zu verfolgen. Es war zu erwarten, daß auch hier die Natur die Hauptknoten und Hauptzonen aufzeichnen, deren Rangordnung und die Entwicklungswege des Formensystems angeben werde.

Aus den Krystallen wurden durch Mechaniker P. Stoë in Heidelberg Kugeln geschliffen und nachdem einige Vorversuche gezeigt, daß der Weg zum Ziel führt, wurde das Studium der Wachstumskörper aus Kugeln im Herbst 1909 in Angriff genommen. Die Versuche sind noch im Gang. Sie liefern schöne Resultate, die später ausfübrlich mitgeteilt werden sollen.

Schon 1895 hat A. Rauber ${ }^{1}$ ) in Dorpat Kugeln wachsen lassen und die Resultate seiner Züchtungen in prächtigen Photographien publiciert. Er war als Mediciner nicht in der Lage, die krystallographischen Methoden auf die Producte anzuwenden. Von den Krystallographen andererseits sind die Publicationen Rauber's wenig beachlet worden.

Indessen zeigten Studien am Diamant mit A. v. Fersmann, daß die krummen Formen dieses merkwürdigen Minerals als Lösungsgebilde aufzufassen sind; die ebenflächigen und scharfkantigen als Wachstumsgebilde; daß aber die meisten Diamantkrystalle Wachstums- und Lösungsgebilde zugleich aufweisen. Und zwar zeigten sich bei solchen Krystallen (wir nannten sie neutrale) die Oktaẹderflächen $(p)$ wachsend, während Würfel (c) und Dodekaëder (d) sich lösten.

Es ergab sich folgende Erklärung der Erscheinungen: Der wachsende Diamantkrystall nimmt aus der übersättigten Mutterlauge (Magma) den überschüssigen Kohlenstoff weg, solange bis das Magma neutral ist, das heißt bis es dem Krystall Substanz weder gibt noch nimmt. Ist nun die Fähigkeit zum Anlagern nach verschiedenen Richtungen verschieden (was bei allen Krystallarten der Fall sein dürfte), so ist eine für den Krystall als Ganzes neutrale Lauge nicht neutral für alle Richtungen (Knoten, Flächenarten). Sie ist für die eine übersättigt, wenn sie für die andere neutral oder untersättigt ist.

1) Die Regeneration der Krystalle, Leipzig 1895 und 1896 . Ausz. diese Zeitschr. 29, 409. - Atlas der Krystallregeneration, Dorpat 1897. 
Somit bringt eine neutrale Lauge Wachstums- und Lösungserscheinungen zugleich hervor (wie wir dies beim Diamant erkennen), ohne daß die Sättigung der Lauge sich ändert. Es kann von einer Flächenart (z. B. c) Substanz weggenommen und der anderen (z. B. $p$ ) aufgelagert werden.

Schließt ein Krystall seine Bildungsgeschichte in diesem neutralen $\mathrm{Zu}$ stand ab (durch Erstarren des Magmas, durch Abfließen der Mutterlauge, durch Herausnehmen), so zeigt das fertige Gebilde die gemischten Erscheinungen. Wir nennen ihn einen neutralen Krystall $(N)$ im Gegensatz zum wachsenden Krystall $(W)$, der beim Wachsen und zum Lösungskrystall $(L)$, der beim Lösen seine Bildungsgeschichte abgeschlossen hat.

Besonders günstig für die Bildung neutraler Krystalle sind die Umstände, unter denen wir annehmen dürfen, daß der Diamant sich gebildet hat. Eine große Schmelzmasse, in der die Diamantkrystalle sich schwebend ausbildeten, die im Zustand der Neutralität geblieben sein dürfte, bis sie erstarrte.

Eine kleine Veränderung im Magma zugunsten des Lösens (z. B. Erwärmen) läßt die Wachstumserscheinungen verschwinden und macht den Krystall zu einem Lösungsgebilde. Eine kleine Veränderung zugunsten des Wachsens läßt die Lösungserscheinungen verschwinden und macht den Krystall zum reinen Wachstumskörper.

In einem größeren Magma, ja in demselben GefäB sind die Verhältnisse nicht überall die gleichen. So kann es geschehen, daß zur selben Zeit an einer Stelle Lösungsgebilde auftreten, an der anderen Wachstums- und an der dritten neutrale Gebilde, daß an demselben Fundort bei demselben Mineral alle drei Arten von Gebilden angetroffen werden.

Die Entfernung, in der das Ungleiche gleichzeitig entstand, kann klein sein. Ja wir fanden am Diamant Krystalle und Krystallgruppen von wenigen Millimetern Größe, die auf der einen Seite Wachsen, auf der anderen Lösung zeigen. Hier dürften Nachbarn und Strömungen bestimmend mitgewirkt haben.

Studien am Topas (mit V. Rosický), die gleichzeitig mit den Diamantstudien geführt wurden, bestätigten und ergänzten das am Diamant Gefundene. Auch beim Topas zeigten sich bei vielen Krystallen, wenn nicht bei den meisten, Wachstums- und Lösungserscheinungen gemischt. Auch hier treffen wir neutrale Krystalle in großer Zahl neben reinen Wachstumskrystallen. Auch da finden wir gewisse Flächenarten für das Wachsen, andere für das Lösen bevorzugt.

Es wurde dann bei anderen Krystallarten Umschau gehalten und es zeigte sich die Erscheinung der neutralen Gebilde neben den reinen Wachstumsgebilden als eine weit verbreitete. Man kann sagen als eine 
allgemeine, in dem Sinn, daß jede Krystallart ihre neutralen Krystalle hat, wenn sie auch noch nicht für alle nachgewiesen sind.

Reine Lösungskörper sind bei den natürlichen Krystallen selten, aber sie kommen vor. Man kennt sie außer beim Diamant, beim Beryll, Castor, Pollux, Spodumen, Flußspat und anderen. Die Ursache der größeren Seltenheit dürfte darin zu suchen sein, daß, wenn der Lösungsproceß alle Flächenarten angreift, in der Regel der ganze Krystall aufgezehrt wird.

Es wurden nun am Diamant Lösungsversuche gemacht, um zu prüfen, ob die Deutung der natürlichen Gebilde mit dem Experiment in Einklang steht. Eine solche Übereinstimmung hat sich in der Tat ergeben.

Leider war es nicht möglich, den Diamant oder den Topas wachsen zu lassen und dadurch neutrale Gebilde für sie zu erhalten. Es erschien daher wünschenswert, das Studium der neutralen Gebilde experimentell bei solchen Krystallarten durchzuführen, bei denen es möglich ist, das heißt, bei denen man das Wachsen und Lösen leicht herbeiführen und beeinflussen kann. Besonders geeignet sind solche, aus denen man genügend große Krystalle züchten kann, um aus ihnen Kugeln zu schleifen.

Als erste Beispiele wurden gewählt: Alaun, Kupfervitriol, Nickelvitriol, Salmiak. Der Alaun, weil er der Form nach dem Diamant ähnlich ist und sich erwarten ließ, daß unmittelbar Vergleichbares auftreten werde. Das hat sich auch bewährt. Der Kupfervitriol als triklines Beispiel, der Nickelvitriol als rhombisches Beispiel. Bei ihm und seinen Verwandten sollten die durch die Isomorphie bewirkten Erscheinungen studiert werden. Als tetragonales Beispiel wurde das gelbe Blutlaugensalz zugefügt.

Die Versuche an der Kugel, sowie an Krystallen mit ebenen Flächen sind im Gang und es soll über die Resultate später ausführlich berichtet werden.

Heidelberg, August 1910. 